\title{
EFFECT OF CLEANING AND DISINFECTION ON NATURALLY CONTAMINATED CLINICAL CONTACT SURFACES
}

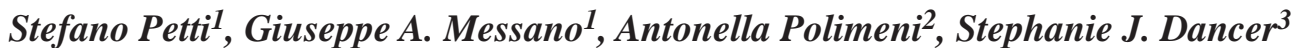 \\ ${ }^{1}$ SAPIENZA UNIVERSITY, DEPARTMENT OF PUBLIC HEALTH AND INFECTIOUS DISEASES, ROME, ITALY; \\ ${ }^{2}$ SAPIENZA UNIVERSITY, DEPARTMENT OF ORAL AND MAXILLOFACIAL SCIENCES, ROME, ITALY; \\ ${ }^{3}$ HAIRMYRES HOSPITAL, DEPARTMENT OF MICROBIOLOGY, EAST KILBRIDE LANARKSHIRE, UK
}

\begin{abstract}
Introduction. Data regarding methicillin-resistant Staphylococcus aureus, Legionella pneumophila, Hepatitis B virus suggest that the environment plays an important role in infection transmission in dental healthcare settings. The Centres for Disease Control and Prevention suggest covering clinical contact surfaces with disposal barriers or disinfecting them between patients. Both methods have drawbacks; preliminary data suggest that cleaning could be an alternative.

Aim. To investigate microbial contamination on dental chairs after dental therapy with turbine and decontamination power of disinfection and cleaning.

M aterial and methods. We assessed microbial contamination of a dental chair, used by paediatric patients receiving conservative/endodontic therapy and located in an annex isolated from the rest of the dental office. Contact plates containing Nutrient Agar were used to assess microbial load, immediately before and after treatment, and following one of two decontamination protocols. Protocol-1 comprised cleaning (sodium lauryl sulphate-based soap) followed by disinfection (hypochlorite solution); and protocol-2 was soap-based cleaning only. Contamination levels were classified as no growth; scanty $(<2.5 \mathrm{CFU} /$ cm2); light (2.5-12 CFU/cm2); moderate (12-40 CFU/cm2); or heavy growth (40-100 CFU/cm2).

Results. Contamination ranged between moderate and heavy growth for 93.3\% samples after dental therapy before decontamination. Scanty growth was obtained from $93.3 \%$ samples and $96.7 \%$ samples after protocol 1 and protocol-2, respectively. Initial level of contamination had no significant effect on the final level and the decontamination power of the two protocols was not significantly different either.

Conclusion. Dental therapy produced high levels of microbial contamination which justified the use of adequate disinfection and/or cleaning. Cleaning alone was sufficient to decontaminate the surface of the dental chair, while disinfection offered no additional effect.
\end{abstract}

Key words: Infection Control, Dentistry, Clinical Contact Surface, Environmental Contamination, Healthcare-Associated Infections

\section{Introduction}

Infection control in dentistry is aimed at reducing the occupational risk of infection among dental healthcare workers and the risk of healthcare-associated infections among patients. The appropriateness of infection control measures is assessed and modelled according to the available scientific evidence, but data regarding ascertained cases of infection in dental healthcare settings are sparse ${ }^{1}$. Currently, there are two lines of reasoning leading to guidelines for infection control in dentistry. According to the more flexible of these lines, preventive measures are based on reports of infections directly attributable to identified sources of transmission. The underlying principle of these guidelines is that infection control procedures cannot eradicate infection transmission, but can realistically seek to reduce the excess risk of infection to an acceptable level. The term acceptable risk, which evolved from the realization that absolute safety is an unachievable goal, describes the likelihood of an event whose probability of occurrence is small ${ }^{2}$. A ccording to the more severe line of reasoning, the few ascertained cases of infection occurred in dental healthcare settings do not necessarily mean that the infection risk is minimal, but could be due to insufficient evidence, thus leaving room for the application of the precautionary principle, which states that when an activity presents an uncertain potential for substantial harm to human health, precautionary measures

(C) 2013 Faculty of Medicine in Niš. Clinic of Dentistry in Niš. All rights reserved / (C) 2013 Medicinski fakultet Niš. Klinika za stomatologiju Niš. Sva prava zadržana
Address for correspondence:

Prof.Dr. Stefano Petti

Department of Public Health and Infectious Diseases

Sapienza University

P.le Aldo Moro 5, 00185 Rome, Italy

Phone/Fax: +3906 49914667

Email: stefano.petti@uniroma1.it 
should be taken even if there is no scientific evidence that such measures are needed or effective ${ }^{3}$. Guidelines based on the acceptable risk seek to decrease the occupational risk, the risk of infection for patients and, indirectly, the risks of litigation and dental treatment failure due to surgical site infection. Such guidelines are supposed to increase awareness and achieve high level of consensus from dental healthcare workers. Guidelines based on the precautionary principle seek to produce a checklist of procedures and processes. These checklists are practical and applicable by inexperienced staff, but they could make dental healthcare workers less aware of which dental procedures yield the highest risk of transmission, because they may be received but not necessarily understood by dental healthcare workers ${ }^{4}$.

The ascertained cases of serious infections in dental healthcare settings, such as hepatitis B, AIDS and tuberculosis, are generally dateD before the 90 's, when universal/standard precautions were not produced and dental healthcare workers' awareness toward infection transmission was low, if not lacking at all. The guidelines released by the Centers for Disease Control and Prevention (CDC) in 1986 were the first attempt to rationalize the infection control practices. These guidelines were essentially based on the precautionary principle and the Authors explicitly admitted that, "The assessment of quantifiable risks to dental personnel and patients for specific diseases requires further research"5. Improved knowledge regard- ing infection transmission in dentistry, led to updated infection control guidelines in 1993 which adopted "specific strategies directed to the prevention of transmission of pathogens among dental healthcare workers and their patients" ${ }^{\prime \prime}$. The last guideline update of 2003, decreased the importance of precautionary principle in favour of the acceptable risk principle ${ }^{1}$. After 2003, several important cases of infection in dentistry occurred, such as one case of hepatitis B transmitted between patients ${ }^{7}$ and one case of legionellosis ${ }^{8}$, while the risk from nosocomial pathogens, such as methicillin-resistant Staphylococcus aureus (MRSA) ${ }^{9}$ and al ternative routes of opportunistic pathogen transmission, such as dental unit waterlines ${ }^{10,11}$, were reported.

These data suggest that the environment could be a potential reservoir of nosocomial and opportunistic pathogens -characterized by long-term survival on non-biological substrates $^{12}$, which could be transmitted between patients and staff. Most data regarding the role of the environment come from hospitals, where this risk is the highest because invasive surgical procedures are frequently made, patients are often immune-compromised and overcrowding frequently occurs. Infections caused by microorganisms, such as M RSA, vancomycin-resistant enterococci (VRE), Clostridium difficile, Acinetobacter spp, Norovirus, etc. are promoted by contaminated surfaces and equipment ${ }^{13-16}$ (Figure). In absence of further data regarding

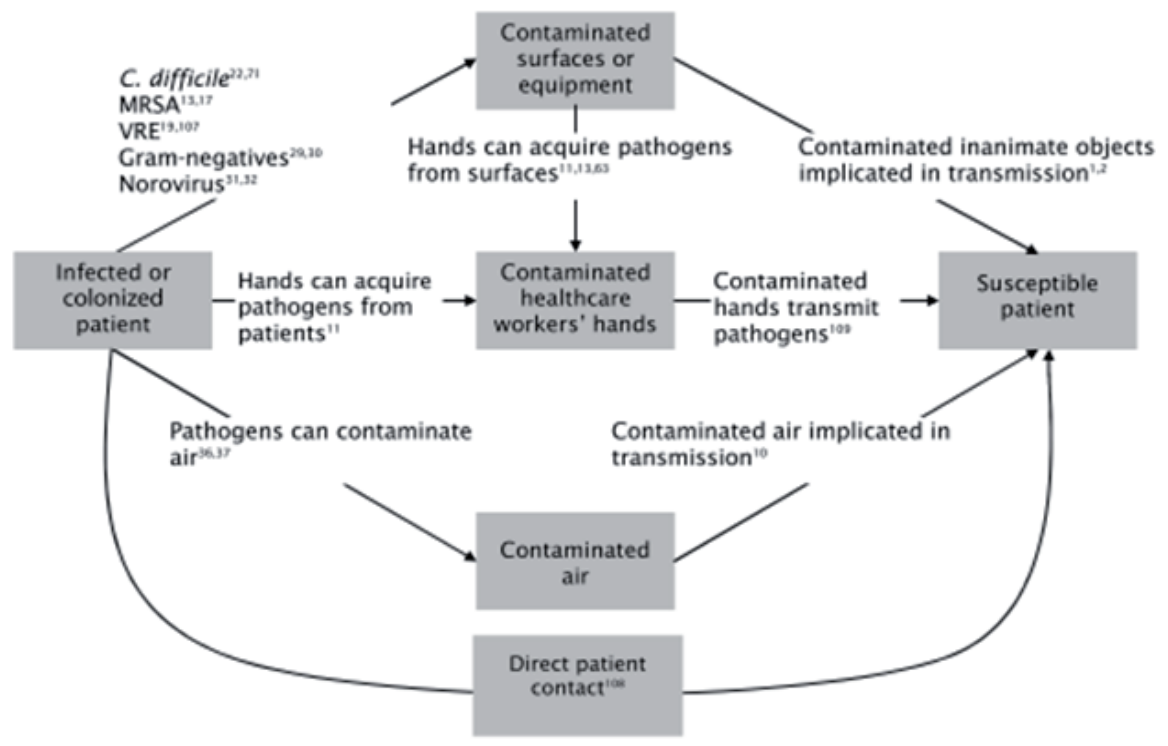

F igure 1. Generic transmission routes of nosocomial pathogens through the environment (contaminated surfaces or equipment). MRSA: methicillin-resistant Staphylococcus aureus; VRE: vancomycin-resistant enterococci. From ref. [16-Otter JA, Yezli S, French GL. The role played by contaminated surfaces in the transmission of nosocomial pathogens. Infect Control Hosp Epidemiol 2011;32(7):687-99.]. 
the role of the environment in dental heal thcare settings, infection-control practices directed at clinical contact surfaces (i.e. surfaces contaminated from patient materials by direct aerosol/ splatter generated during dental procedures and touched by dental healthcare workers) require justification. The Centres for Disease Control recommend use of tuberculocidal disinfectants or to protect surfaces with disposal barriers ${ }^{1}$. Cleaning could be a valid alternative. Indeed, disposable barriers, cleaning with soap, and disinfection with hypochlorite produced the same level of effectiveness in controlling MRSA on artificially contaminated dental chairs ${ }^{17}$.

\section{Aim}

The aim of this study was to investigate the effect of cleaning and disinfection in reducing the microbial load from dental chairs naturally contaminated after therapy of patients using the turbine.

\section{Material and M ethods}

\section{Setting}

The clinical contact surface chosen was a dental chair covered with skai upholstery. This chair was located in an annex of a dental department of the Sapienza University of Rome, where routine clinical activity is performed. The annex had natural and artificial light/ventilation and was not sealed from the rest of the unit: the walls were transparent and open at the top. The area of the annex was $16 \mathrm{~m}^{2}$. Air speed, temperature, and humidity, measured during a previous study made between September and July, ranged between 0.00 and $0.03 \mathrm{~m} / \mathrm{s}, 18.7^{\circ} \mathrm{C}$ and $25.9^{\circ} \mathrm{C}$, and $22.9 \%$ and $47.1 \%$, respectiveIy ${ }^{12}$. From patient to patient and at the end of each working session, environmental surfaces were subjected to daily cleaning and disinfection procedures following CDC guidelines ${ }^{1}$. The study protocol was approved by the board of the department and the study performed between 2012 and 2013.

\section{Microbiological assessment}

At every testing occasion, a dental patient underwent treatment which required extensive use of the turbine. In order to prevent additional contamination, dental healthcare workers wore single-use uniforms, gloves, mask and protective glasses before entering the annex and removed them only at the end of the testing occasion. Before the patient was admitted to the annex, environmental samples were performed using five Replicate Organism Detection and Counting (Rodac) plates (Becton Dickinson Italia, Buccinasco, Italy) containing Nutrient Agar (Becton Dickinson Italia) and following standard procedures. Briefly, plates were pressed on to surfaces for $30 \mathrm{~s}$ at a pressure of $20-25 \mathrm{~g} / \mathrm{cm}^{2}$ and incubated in air at $30^{\circ} \mathrm{C}$ for 48 h. Contact plates were preferred to swabs because they provide quantifiable and repeatable results from environmental surfaces ${ }^{18}$. M icrobial growth was then quantified and expressed in colony forming units $(\mathrm{CFU}) / \mathrm{cm}^{2}{ }^{19}$. The area tested, between the seat and the sides, is frequently touched by patients during therapy and the plates were pressed against different sites of this area.

After screening the dental chair, the patient was admitted to the annex and treated. Immediately after therapy, the patient left the annex and post-treatment screening was performed. The microbial load detected on the dental chair due to the dental therapy was assessed using the difference between the two screens.

After the second sample, decontamination was performed following two different protocols.

- Protocol-1: disinfection (wipe-rinse method). A new cloth was washed with tap water to decrease initial rigidity and soaked with 20$40 \mathrm{~mL}$ of tap water. 1-3 mL of commercially available soap containing 5-10\% sodium lauryl sulphate (SLS) was placed on the soaked cloth. The cloth was used for 1-1.5 min with rotational movements on the contaminated surface. Then it was rinsed with tap water and soaked with $10-20 \mathrm{~mL}$ of a $1: 10$ dilution of $5.25 \%$ to $6.15 \%$ sodium hypochlorite. The cloth was used with similar movements on the contaminated surface for the same time. The solution remained on the upholstery for $7 \mathrm{~min}$. The cloth was thoroughly washed, soaked with tap water and used with the same movements and modalities on the upholstery for 3 min to remove hypochlorite residuals. The latter procedure was repeated twice. 
- Protocol-2: cleaning. A new cloth was washed with tap water to decrease initial rigidity and soaked with $20-40 \mathrm{~mL}$ of tap water. 1-3 $\mathrm{mL}$ of soap was placed on the soaked cloth, which was used for 1-1.5 min with rotational movements on the contaminated surface. The cloth was washed, soaked with tap water and used with the same movements and modalities on the upholstery for $3 \mathrm{~min}$ to remove SLS residuals, which may yield antibacterial activity. The latter procedure was repeated twice.

At every testing occasion, only one protocol was adopted. Protocols were alternated. After the decontamination process, a third series of environmental screening samples was collected following the same procedures ${ }^{17}$.

The level of the microbial density on the dental chair was classified as no growth; scanty $(<2.5 \mathrm{CFU} / \mathrm{cm} 2)$; light $(2.5-12 \mathrm{CFU} / \mathrm{cm} 2)$; moderate $(12-40 \mathrm{CFU} / \mathrm{cm} 2)$; or heavy growth (40-100 CFU/cm2). Aerobic colony counts on hand-touch sites after decontamination should not exceed 2.5-5 CFU $/ \mathrm{cm}^{2} 19$.

The study was performed with the first patient of the working session and only one testing occasion occurred each day. Every protocol was tested thirty times, with a total of sixty testing occasions.

\section{Statistical analysis}

M ean microbial densities before decontamination and after the two protocols were assessed with $95 \%$ confidence intervals (95CIs), differences between mean densities before and after each protocol were statistically analysed with the Student's t-test for paired samples, while for the differences between mean densities with protocol-1 and protocol-2 the Student's t-test for unpaired samples was used. The relative decrease in microbial density due to decontamination was assessed by the formula: " $\{1-[$ (density after decontamination)/(density before decontamination) $\} \times 100$ ". The mean relative decreases and 95CIs using the two protocols were assessed and compared with the Student's t-test for unpaired samples.

The level of microbial densities before and after the decontamination were classified as no growth, scanty, light, moderate, and heavy growth. In addition, density levels after the decontamination were classified into acceptable (density, $\leq 2.5 \mathrm{CFU} / \mathrm{cm} 2$ ) and unaccept- able (density, $>2.5 \mathrm{CFU} / \mathrm{cm}^{2}$ ), choosing the most severe threshold level for acceptability ${ }^{19}$. The distributions of the densities according to their levels were calculated and the differences within each protocol between before and after decontamination and between protocols were assessed with the $\chi 2$ test.

Finally, the difference in the decontamination power between the two protocols accounting for the baseline contamination level was investigated, as it was assumed that the final microbial density was affected by the initial density and not only by the type of decontamination protocol. Therefore, the microbial density after the decontamination was regressed upon the density before the decontamination and the residuals were estimated. R esiduals can broadly be considered as the microbial densities after the decontamination adjusted for the level of density before the decontamination. The Student's t-test for unpaired samples was then used to compare the means of residuals with the two protocols. For all the analyses, the level of significance was set at $95 \%$.

\section{Results}

Patients received caries treatments, fiftyone conservative interventions (cavity preparation and filling placement) and nine endodontic interventions (pulpectomy). Rubber dam was not used, while dental vacuum suction was active throughout all the intervention. $N$ one of the treated children developed infections at the site of local anaesthetic injection or at the treated site.

The microbial density detected on the skai upholstery of the dental chair soon after therapy with dental turbine ranged between 8.6 and 41.6 $\mathrm{CFU} / \mathrm{cm}^{2}$. The mean microbial densities before the two decontamination protocols were 27.5 $\mathrm{CFU} / \mathrm{cm}^{2}$ (before disinfection) and $24.5 \mathrm{CFU} /$ $\mathrm{cm}^{2}$ (before cleaning), the difference between initial densities was not significant, thus showing that the starting conditions were similar for both protocols (Table 1). The decontamination protocols led to $97.5 \%$ and $96.6 \%$ decreases in density with protocol-1 (disinfection) and protocol-2 (cleaning), leading to final mean densities of 0.7 and $0.8 \mathrm{CFU} / \mathrm{cm}^{2}$, respectively. The differences between the two protocols were not significant. 
Table 1. Mean microbial densities and mean relative density decreases before and after decontamination (protocol-1: cleaning with sodium lauryl sulphate and disinfection with hypochlorite; protocol-2: cleaning with sodium lauryl sulphate). Statistical analysis of differences with Student's t-test.

\begin{tabular}{lll}
\hline & Mean density $-\mathrm{CFU} / \mathrm{cm} 2-$ & Mean relative decrease -\% \\
\hline Before protocol-1 & $27.50(24.71-30.26)^{\mathrm{a}, \mathrm{c}}$ & \\
After protocol-1 & $0.70(0.35-1.04)^{\mathrm{b}, \mathrm{c}}$ & $97.5(96.3-98.7)^{\mathrm{e}}$ \\
Before protocol-2 & $24.49(21.71-27.26)^{\mathrm{a}, \mathrm{d}}$ & \\
After protocol-2 & $0.76(0.48-1.04)^{\mathrm{b}, \mathrm{d}}$ & $96.6(95.2-98.0)^{\mathrm{e}}$ \\
\hline
\end{tabular}

$a_{t}=1.50 ; p=0.14 ;{ }^{b}=0.28 ; p=0.78 ;{ }^{c}=19.28 ; p<0.0001 ;{ }^{d}=17.02 ; p<0.0001 ;{ }^{e}=0.96 p=0.34$

Table 2. Levels of microbial density before and after the two decontamination protocols (protocol-1: cleaning with sodium lauryl sulphate and disinfection with hypochlorite; protocol-2: cleaning with sodium lauryl sulphate). Statistical analysis of differences with $\chi^{2}$ test.

\begin{tabular}{lllll}
\hline & Scanty (acceptable) & Light & Moderate & Heavy \\
\hline Before protocol-1 & $0.0 \% \%^{\mathrm{a}}$ & $3.3 \% \mathrm{a}$ & $93.3 \%^{\mathrm{a}}$ & $3.3 \%^{\mathrm{a}}$ \\
After protocol-1 & $93.3 \%^{\mathrm{b}}$ & $6.7 \%^{\mathrm{b}}$ & $0.0 \%^{\mathrm{b}}$ & $0.0 \%^{\mathrm{b}}$ \\
Before protocol-2 & $0.0 \%^{\mathrm{a}}$ & $10.0 \% \mathrm{a}$ & $90.0 \%^{\mathrm{a}}$ & $0.0 \%^{\mathrm{a}}$ \\
After protocol-2 & $96.7 \%^{\mathrm{b}}$ & $3.3 \% \mathrm{~b}$ & $0.0 \%^{\mathrm{b}}$ & $0.0 \%^{\mathrm{b}}$ \\
\hline
\end{tabular}

${ }^{a} \chi 22 d f=2.02 ; p=0.36 ;{ }^{b} \chi 21 d f=0.35 ; p=0.55$

The density level before decontamination was generally moderate between 12 and $40 \mathrm{CFU} / \mathrm{cm}^{2}$, excluding one case (before protocol-1, 3.3\% of all tests) of heavy microbial density (Table 2). The distributions of densities before decontamination with the two protocols were non-significantly different, suggesting that disinfection and cleaning were assessed starting from similar contamination levels. A fter decontamination, microbial density was scanty, that is, acceptable in $93.3 \%$ (protocol-1) and $96.7 \%$ (protocol-2) of tests. In three cases, the decontamination protocols led to unacceptable levels of microbial density. However, these densities were only marginally higher than the chosen threshold level of $2.5 \mathrm{CFU} / \mathrm{cm} 2$, as they were 2.6 and $3.9 \mathrm{CFU} / \mathrm{cm} 2$ (after disinfection) and $3.1 \mathrm{CFU} / \mathrm{cm} 2$ (after cleaning).

The microbial densities after decontamination were then regressed upon the microbial densities before decontamination and the residuals were estimated; means for protocol-1 and protocol-2 were calculated and the difference between protocols was statistically analysed. The t-test value was $0.58(\mathrm{p}=0.56)$ (data not in Table). These results denote that the microbial density resulting after the decontamination was only minimally affected by the baseline microbial density produced by the dental therapy and that the effect of the two decontamination protocols was similar.

\section{Discussion}

The present study is one of the papers presented at the workshop "Advances in Infection Epidemiology and Control in Dental Healthcare Settings", Department of Public Health and Infectious Diseases, Sapienza University, Rome, Italy on February 9th, 2013 20-26.

The nonspecific contamination level of the dental chair after therapy with dental turbine was, on average, moderate. Such a level is probably different for different types of dental treatments. For example, professional toothcleaning with ultrasonic scaler or surgical extractions could produce heavy contamination, while sealant placement or orthodontic treatment could produce light contamination. Low contamination levels after dental therapy were reported in other studies from Italy. The clinical contact surface under consideration was the tray in front of patients. Authors reported that microbial density level was lower than $1 \mathrm{CFU} /$ $\mathrm{cm}^{2}$ in $54 \%$ cases and mean density was 1.3 $\mathrm{CFU} / \mathrm{cm}^{2}{ }^{27}$. In a similar study, density lower than $1 \mathrm{CFU} / \mathrm{cm} 2$ was reported in $80 \%$ cases and mean density was $0.6 \mathrm{CFU} / \mathrm{cm}^{2} 28$. The reported values after dental therapy suggest that in the majority of daily activity, dental therapy probably produces a minimal level of environmental contamination, which would be defined as scanty and therefore acceptable even before the decontamination process.

A Iternatively, these data could suggest that non-specific microbiological assessment of environmental contamination could be mislead- 
ing. Indeed, data on nosocomial pathogens, such as MRSA, suggest that transmission through the environment is possible, although unlikely, in dental healthcare settings. For example, eight out of 140 patients in a ward of a department of special dental care and oral surgery in Japan became MRSA carriers during hospitalization. Environmental decontamination procedures and use of disposal barriers were sufficient to prevent further MRSA dissemination ${ }^{29}$. Another survey from US reported that $21 \%$ dental students and $8 \%$ environmental surfaces were positive for MRSA and in one case, the strain sampled from the dental chair was the same as that detected from one student, suggesting that either the students acquired the microorganism from the environment or vice versa ${ }^{30}$. Studies on methicillin-susceptible S. aureus (MSSA) reported that its detection in the environment is frequently higher in non-operative than operative areas. For example, in a paediatric dental clinic in Brazil, airborne contamination resulted more than three times higher in storerooms than in dental boxes where dental treatments were made. In this case, patients wore rubber dam to decrease environmental contamination and this measure was enough to control for M SSA environmental contamination ${ }^{31}$. A ccording to a survey from UK, airborne MSSA levels were higher in reception and other non-clinical areas than in clinical areas ${ }^{32}$. A survey from a dental school in US found that environmental contamination by MSSA was almost double outside than inside patient care areas $(4.7 \% \text { vs. } 3.7 \%)^{33}$.

A $n$ interesting study on hand-touch surfaces (bedside lockers, bedrails, overbed table) from an Scottish hospital reported that cleaning performed by trained personnel may decrease the nonspecific microbial load to levels below the threshold of $5 \mathrm{CFU} / \mathrm{cm} 2$ for up to 24 hours. A similar decrease was reported for MSSA, while MRSA were only occasionally detected before and after cleaning ${ }^{34}$.

These data collectively suggest two important findings. Firstly, environmental contamination is more likely to be due to non-specific contamination arisen by everyday activity such as touching, talking, changing clothes, etc., than to dental practice. Secondly, generic contamination is not necessarily suggestive of the risk for infection by nosocomial pathogens and the occasional assessment of specific contamination by these micro-organisms could be useful to assess the infection risk from environmental surfaces.

Despite the reported low levels of environmental contamination, the control of microbial contamination of clinical contact surfaces remains important. CDC proposed to make this control using disposal barriers or disinfecting contaminated surfaces between patients ${ }^{1}$. Both methods are not free from drawbacks. Indeed, the number and amount of clinical contact surfaces to cover with disposable barriers and the number of times barriers must be changed during a working day are so many that this measure is costly and impractical. In addition, barrier materials create an important environmental impact ${ }^{35}$. On the other hand, there are serious concerns regarding costs and toxic effects of disinfectants on cleaning staff and environment ${ }^{36}$. The third option, that is, cleaning, could help minimize the impact on the environment and staff and without decreasing the effectiveness in removing microbial contamination from clinical contact surfaces. There are few data regarding the differences between cleaning and disinfection in terms of control of infection transmission in dentistry. The only study performed was centred on MRSA and reported that disposal barriers, disinfection with hypochlorite and cleaning with SLS provided similar results in terms of ability to remove artificial MRSA contamination from a dental chair ${ }^{17}$. Another study on formica surfaces reported that cleaning, disinfection with a disinfectant with sporicidal activity and cleaning with the same disinfectant yielded similar ability to decrease the number of spore of Clostridium difficile starting from a high contamination level of 104-105 CFU ${ }^{37}$. However, the crucial point of an effective method to control for environmental microbial contamination is not the number of microorganisms that the method can remove, but the decrease of dental heal thcare associated infection rate which is attributable to the decontamination procedure.

Studies of this type are scarce in the literature, including other settings where healthcare associated infections are more frequent and serious, such as in intensive care units of hospitals. Systematic reviews on hospital settings report inconclusive results for both disinfection ${ }^{38}$ and cleaning ${ }^{36}$, as some studies do report no decrease in infection rates, while other studies report a significant decrease attributable to the 
decontamination protocol under investigation. Thus, even in settings at high risk for infections transmitted through the environment, the effectiveness of cleaning is not higher than the effectiveness of disinfection and vice versa.

\section{Conclusion}

In conclusion, the level of microbial contamination of a clinical contact surface produced during dental therapy with turbine is generally moderate $\left(12-40 \mathrm{CFU} / \mathrm{cm}^{2}\right)$. Although in the present study no infection was associated with this level of contamination, these data do not suggest that environmental infection control procedures should be abandoned, particularly when we know that in certain healthcare settings few CFUs of nosocomial pathogens may be sufficient to initiate infection. The study also did not find any difference between disinfection with hypochlorite and cleaning with SLS containing soap in ability to remove microorganisms from contaminated dental chairs following routine dental care. 


\section{LITERATURA / REFERENCES}

1. K ohn W G, Collins AS, Cleveland JL et al. Guidelines for infection control in dental health-care settings, 2003. M M W R Recomm Rep 2003;52(RR-17):1-61.

2. Petti S, Polimeni A. The rationale of guidelines for infection control in dentistry: precautionary principle or acceptable risk? Infect Control Hosp Epidemiol 2010;31(12):1308-10.

3. Laheij AM, Kistler JO, Belibasakis GN, Välimaa H, de Soet JJ; European Oral Microbiology Workshop (EOMW) 2011 Healthcare-associated viral and bacterial infections in dentistry. J Oral Microbiol 2012;4. doi: 10.3402/jom.v4i0.17659.

4. Petti S. Why guidelines for early childhood caries prevention could be ineffective amongst children at high risk. J Dent 2010;38(12):946-55.

5. Centers for Disease Control. Recommended infectioncontrol practices for dentistry. MMWR Morb Mortal Wkly Rep 1986;35(15):237-42

6. Centers for Disease Control. 1993. Recommended infection-control practices for dentistry. MMWR Recomm Rep 1993;42(RR-8): $1 \$ 12$

7. Redd JT, Baumbach J, Kohn W, Nainan O, Khristova M, Williams I. Patient-to-patient transmission of hepatitis B virus associated with oral surgery J Infect Dis 2007;195(9):131114.

8. Ricci ML, Fontana S, Pinci F et al. Pneumonia associated with a dental unit waterline. Lancet 2012;379(9816):684

9. Petti S, Polimeni A. Risk of methicillin-resistant Staphylococcus aureus transmission in the dental healthcare setting: a narrative review. Infect Control Hosp Epidemiol 2011;32(11):1109-15.

10. O’Donnell MJ, Boyle MA, Russell RJ, Coleman DC Management of dental unit waterline biofilms in the 21 st century. Future Microbiol 2011;6(10):1209-26

11. Petti S, Moroni C, Messano GA, Polimeni A. Detection of oral streptococci in dental unit water lines after therapy with air turbine handpiece: biological fluid retraction more frequent than expected. Future Microbiol 2013;8(3):413-21.

12. Petti S, De Giusti M, Moroni C, Polimeni A. Longterm survival curve of methicillin-resistant Staphylococcus aureus on clinical contact surfaces in natural-like conditions Am J Infect Control 2012;40(10):1010-2

13. Boyce JM. Environmental contamination makes an important contribution to hospital infection. J Hosp Infect 2007;65(Suppl 2):50-4.

14. Dancer SJ. The role of environmental cleaning in the control of hospital-acquired infection J Hosp Infect 2009;73(4):378-85.

15. Weber DJ, Rutala WA, Miller MB, Huslage K, Sickbert -Bennett E. Role of hospital surfaces in the transmission of emerging health care-associated pathogens: norovirus, Clostridium difficile, and Acinetobacter species. Am J Infect Control 2010;38(5 Suppl 1):S25-33

16. Otter JA, Yezli S, French GL. The role played by contaminated surfaces in the transmission of nosocomial pathogens Infect Control Hosp Epidemiol 2011;32(7):687-99.

17. Petti S, Polimeni A, Dancer SJ. Effect of disposable barriers, disinfection, and cleaning on controlling methicillinresistant Staphylococcus aureus environmental contamination. Am J Infect Control 2013. doi:pii: S0196-6553(12)01270-9.

18. Galvin S, Dolan A, Cahill O, Daniels S, Humphreys $\mathrm{H}$. Microbial monitoring of the hospital environment: why and how? J Hosp Infect 2012;82(1):143-151.

19. Mulvey D, Redding $\mathrm{P}$, Robertson $\mathrm{C}$ et al. Finding a benchmark for monitoring hospital cleanliness. J Hosp Infect 2011;77(1):25-30.

20. Messano GA, Sofan AAA, Petti S. Quality of air and water in dental healthcare settings during professional toothcleaning. A cta Stomatol Naissi 2013; 29(67): 1230-35; doi 10.5937 /asn1367230M .
21. Messano GA, Masood M, Palermo P, Petti S. Predictors of L egionella occurrence in dental unit waterlines of a highly colonized dental hospital. Acta Stomatol Naissi 2013; 29(67): 1236-41; doi: 10.5937/asn1367236M.

22. Messano GA, Masood M, Palermo P, Petti S. Prevalence of reactive tuberculin skin test in dental healthcare workers and students. A cta Stomatol Naissi 2013; 29(67): 1242-48; doi: 10.5937/asn1367242M.

23. Petti S, Sofan AAA, Messano GA. Streptococcus pneumoniae carriage rate in healthy preadolescent dental patients. Acta Stomatol Naissi 2013; 29(67): 1249-54; doi: 10.5937/ asn1367249P.

24. Messano GA, De Bono V, Architrave R, Petti S. Environmental and gloves' contamination by staphylococci in dental healthcare settings. A cta Stomatol Naissi 2013; 29(67): 125559; doi: 10.5937/asn1367255M.

25. Messano GA. Bacterial and fungal contamination of dental hygienists' hands with and without finger rings. Acta Stomatol Naissi 2013; 29(67): 1260-64; doi: 10.5937/asn1367260M.

26. Petti S, Messano GA, Scully C. Antibody level and immunity against Hepatitis B virus infection among general dental practitioners. Acta Stomatol Naissi 2013; 29(67): 1273-78; doi: 10.5937/asn1367273P

27. Castiglia P, Liguori G, Montagna MT et al. Italian multicenter study on infection hazards during dental practice: control of environmental microbial contamination in public dental surgeries. BMC Public Health 2008;8:18.

28. Guida M, Gallé F, Di Onofrio V et al. Environmental microbial contamination in dental setting: a local experience. J Prev Med Hyg 2012;53(4):207-12.

29. Kurita $\mathrm{H}$, Kurashina $\mathrm{K}$, Honda T. Nosocomial transmission of methicillin-resistant Staphylococcus aureus via the surfaces of the dental operatory. Br Dent J 2006;201(5):297300.

30. Roberts MC, Soge 00, Horst JA, Ly KA, M ilgrom P. Methicillin-resistant Staphylococcus aureus from dental school clinic surfaces and students- Am J Infect Control 2011;39(8):628-32.

31. Negrini T de C, Duque C, de Oliveira AC, Hebling J, Spolidorio LC, Spolidorio DM. Staphylococcus aureus contamination in a pediatric dental clinic. J Clin Pediatr Dent 2009;34(1):13-8.

32. El-Maaytah M, Drydaki M, Jerjes W et al. Evaluation of airborne contamination with Staphylococcus aureus and MRSA at the Eastman Dental College. Br J Oral Maxillofac Surg 2007;45:e24-5.

33. Trochesset DA, Walker SG. Isolation of Staphylococcus aureus from environmental surfaces in an academic dental clinic. J Am Dent Assoc 2012;143(2):164-9.

34. Bogusz A, Stewart M, Hunter J et al. How quickly do hospital surfaces become contaminated after detergent cleaning? Healthc Infect 2013;18(1):3-9.

35. Rutala WA, Weber DJ. A review of single-use and reusable gowns and drapes in health care. Infect Control Hosp Epidemiol 2001;22(4):248-57.

36. Dancer SJ. Hospital cleaning in the 21st century. Eur J Clin Microbiol Infect Dis 2011;30(12):1473-81.

37. Rutala WA, Gergen MF, Weber DJ. Efficacy of different cleaning and disinfection methods against Clostridium difficile spores: importance of physical removal versus sporicidal inactivation. Infect Control Hosp Epidemiol 2012;33(12):1255-8.

38. Dettenkofer M, Wenzler S, Amthor S, Antes G, Motschall E, Daschner FD. Does disinfection of environmental surfaces influence nosocomial infection rates? A systematic review. Am J Infect Control 2004;32(2):84-9. 